\title{
Gradient-wise search strategy for blind image deblurring
}

\author{
Yunhong Wang*, and Dan Liu \\ Department of Public Security Information Technology and Intelligence Criminal Investigation Police \\ University of China, Shenyang, China
}

\begin{abstract}
Blind image deblurring is a long-standing challenging problem to improve the sharpness of an image as a prerequisite step. Many iterative methods are widely used for the deblurring image, but care must be taken to ensure that the methods have fast convergence and accuracy solutions. To address these problems, we propose a gradient-wise step size search strategy for iterative methods to achieve robustness and accelerate the deblurring process. We further modify the conjugate gradient method with the proposed strategy to solve the bling image deblurring problem. The gradient-wise step size aims to update gradient for each pixel individually, instead of updating step size by the fixed factor. The modified conjugate gradient method improves the convergence performance computation speed with a gradient-wise step size. Experimental results show that our method effectively estimates the sharp image for both motion blur images and defocused images. The results of synthetic datasets and natural images are better than what is achieved with other state-of-the-art blind image deblurring methods.
\end{abstract}

Keywords: Blind image deblurring, Iterative algorithms, Adaptive step size.

\section{Introduction}

\subsection{Retalted work}

In many applications, ranging from computer vision and pattern recognition to machine intelligence, the demand for image deblurring is widely encountered as a prerequisite [1][3]. Often a blurred image needs to be restored to improve its sharpness before further steps, such as segmentation, detection, and classification [4]. Typically, the image deblurring problem can be classified into two categories: non-blind, in which the blur kernel is assumed to be known [5], and blind, in which the blur kernel is unknown [8]-[20]. For nonblind image deblurring (NBID), it is to deblur an image with a known blurring kernel. In a real application, it is usually difficult to know the blurring kernel in advance. Therefore, blind image deblurring (BID) is much more required than NBID. The goal of the BID is to

\footnotetext{
* Corresponding author: wangyunhongcipuc@gmail.com
} 
recover a blur kernel and a sharp latent from a blurred input, which is a typical ill-posed problem. Solution for the ill-posed problem can be categorized into direct methods via some effective regularization [10], [11], [12], [17], [20], [21] and iterative methods via some updating and stopping rules [15], [22]. Recently, learning-based BID approaches have drawn much attention due to the satisfactorily restored qualities [16]. However, note that the effectiveness of the learning-based algorithms highly depends on the training image database, and this kind of algorithm generally requires a high computational complexity. Most recently, significant progresses have been made in[17], [18]. In order to make the blind deblurring problem well-posed, image prior, and blur kernel model exploitation is the key to most effective methods. Well-developed image priors include salient intensity prior [15], hyper-laplacian prior [7], gradient prior [24], surface-aware prior [18], to name just a few. Meanwhile, the blur kernel model includes the depth variation model [26], Forward motion model [27], non uniform model [28]. Most of these methods exploit the image prior and blur kernel model under the Bayesian framework [29]. Even though incorporating regularization to exploit image prior and kernel model helps to increase the probability for achieving a good local solution, the ideal deblurring result cannot be easily acquired.

In this work, we focus on iterative methods by designing a new strategy to improve the comprehensive effect of the BID process. In [14], the alternating direction method of multipliers (ADMM) is employed to solve the proposed model, which has high computational complexity than the general iterative method. However, its applications are constrained due to poor convergence. In [32], [33], Quasi-Newton (QN) method and conjugate gradient (CG) method were proposed to solve the image reconstruction problem. However, the step size was calculated by an inexact line search method that uses a series of iterations to test a suitable value [34]. It requires a large amount of storage and computation and lacks adaptiveness and robustness, performing less satisfyingly with various regularization terms and images, which is less suitable for further application. Therefore, we need to design a more efficient iterative algorithm for BID with suitable step size, reasonable computation cost, and quick convergence speed.

\subsection{Contribution}

Based on the above analysis, we develop several strategies that are significantly different from previous works in the following aspects.

a) A blind image deblurring method is proposed that in troduces a gradient-wise search strategy to calculate a proper step size under an improved BID framework. When dealing with different scenes, the step size adjusts automatically to the change of gradient. It takes less time to generate a more accurate solution with less computation cost. The adaptive iteration method effectively reduces ringing artifacts and increased the fidelity of the deblurred image.

b) A more effective BID framework. It effectively solves the image deblurring model we proposed through combining the conjugate method, which embedded adaptive step size with bilateral total variation (BTV) regularization[30]. The solution space of the latent image is reduced via introduces the BTV regularization, which mainly represents the intrinsic properties of the original image. Furthermore, another useful regularization term can replace the BTV regularization to improve the effiffifficiency with the fundamentally prior information.

Extensive experimental results on blind image deblurring have been provided to evaluate the performance of the proposed method. Compared with other state-of-the-art methods, the results show that this method obtains a superior image quality with less computation cost; qualitative and quantitative comparisons also verify the value of our algorithm. 


\section{Method}

\subsection{Image degradation model}

As an inverse process of image degradation, the BID is tightly dependent on the image degradation model. Let a blurred image having dimension $C^{*} E^{*} \Phi$, where $c=1,2, \ldots$, $C$ are rows, $e=1,2, \ldots, E$ are columns and $\Phi \in\{\operatorname{Re} d$, Green,Blue $\}$. The clear image degrades into a blurred image under the impact of various factors. Therefore, the image degradation process is formulated as follows:

$$
G=W B \otimes I+N
$$

where $G, I$, and $Q$ represent the matrices of the blurred image, clear image, and the additional Gaussian noise acquired during image acquisition; $\otimes$ represents the convolution operator, and $B$ is the blurring kernel. We introduce the warping matrix $W$ as one of the degrading factor considering the real application. Generally, assuming that all low-quality images are generated under the same condition, we can further simplify the model into

$$
G=A \otimes I+N
$$

where $A$ is the total decimation matrix, which has taken all the degrading factors above into consideration. The BID aims to recover the original sharp image from the blurred and warped noisy image without information about the blurring kernel and warping process. We introduce the CG-based BID approach and infer our proposed gradient-wise search strategy. Besides, we adopt $L_{2}$ norm as the fidelity term and employ BTV as the regularization term, and a cost function is always utilized to measure the distance between the estimation and the observation as follows:

$$
\hat{I}=\arg \min _{I} \sum_{l=-p m=0}^{p} \sum^{p} \delta^{|m|+|l|} I-h_{I}^{l} v_{I 1}^{m}+\|G-A I\|_{2}^{2},(m+l \geq 0)
$$

In(3), matrice $h_{I}^{l}$ and $v_{I}^{m}$ shift $I$ by $l$ and $m$ pixels in horizontal and vertical directions, respectively. The scalar weight $\delta(0<\delta<1)$ is applied to the regularization terms to consistent with the actual condition as soon as possible. Considering the computational cost of the conjugate gradient method, we convert $G$ to the $Y C_{b} C_{r}$ color space to obtain the luminance component $Y$ and chromatic components $C_{b}$ and $C_{r}$, give by

$$
\left[\begin{array}{c}
\tilde{Y} \\
\tilde{G}_{b} \\
\tilde{C}_{r}
\end{array}\right]=\left[\begin{array}{ccc}
0.299 & 0.587 & 0.114 \\
-0.172 & -0.339 & 0.512 \\
0.512 & -0.429 & -0.082
\end{array}\right]\left[\begin{array}{c}
G_{r} \\
G_{g} \\
G_{b}
\end{array}\right]
$$

Note that the information related to shape and texture is primarily present in the $Y$ component. We only dispose of the $Y$ component of degraded image $G$, processing $C_{b}$ and $C_{r}$ components with bi-cubic interpolation. Then, we convert the estimated image to the RGB color space. From the discussion above, we rewrite (3) into

$$
\hat{I}_{Y}=\arg \min _{I_{Y}} \sum_{l=-p}^{p} \sum_{m=0}^{p} \delta^{|m|+|l|} I_{Y}-h_{I}^{l} v_{I 1}^{m}+G_{Y}-A I_{Y 2}^{2},(m+l \geq 0)
$$


where $G_{Y}$ and $I_{Y}$ represent the $Y$ component of $G$ and $I$, respectively. To numerically find the solution $I_{Y}$, we use the CG-based method to solve the problem iteratively, and the procedure will be described detailedly in the next part.

\subsection{Proposed algorithm}

After the formulation of the BID model in (5), we can see that estimating a sharp image is equivalent to finding the solution to minimize the cost function $F\left(I_{Y}\right)$. For this minimization problem, we use the CG-based method to obtain a numerical solution, as follows:

$$
\begin{gathered}
\hat{I}_{Y(j+1)}=\hat{I}_{Y(j)}+S_{j} d_{j} \\
d_{j}=-\nabla F\left(I_{Y(j)}\right)+u_{j} d_{j-1} \\
u_{j}=\frac{\nabla F\left(I_{Y(j)}\right)^{\top}\left(\nabla F\left(I_{Y(j)}\right)-\nabla F\left(I_{Y(j-1)}\right)\right)}{d_{j-1}^{\top}\left(\nabla F\left(I_{Y(j)}\right)-\nabla F\left(I_{Y_{j-1}}\right)\right)}
\end{gathered}
$$

where $d_{j}$ and $S_{j}$ represent the descent direction and the step size in the $j$ th iteration, respectively. The current descent direction depends on the former descent direction and the current negative gradient direction. Setting a proper step size plays a vital role in the convergence of the CG method. Since an oversized step size will lead the iteration to a divergence, whereas an undersized step size will cause a slow convergence. Therefore, the optimal step size varies in different scenes, which is challenging to preset appropriately. The Line Search [34] is a widely-used strategy that derives $S_{j}$ by minimizing the objective function as

$$
S_{j}=\arg \min _{S} F\left(I_{Y(j)}+S d_{j}\right)
$$

Although an accurate step size can improve the convergence, the line search procedure cannot quickly solve the large-scaled minimization, and computation cost is expensive. Therefore, we design a gradient-wise search strategy that considering accuracy and computation cost. Firstly, we calculate the first-order derivative of $F\left(I_{Y}\right)$ in (5) as:

$$
\begin{gathered}
\nabla F\left(I_{Y}\right)=-2 A^{\top}\left(G_{Y}-A I_{Y}\right)+\gamma H^{\prime}\left(I_{Y}\right) \\
H^{\prime}\left(I_{Y}\right)=\sum_{l=-p m=0}^{p} \sum_{p}^{p} \delta^{|m|+|l|}\left(Q-h_{I}^{l} v_{I}^{m}\right) \operatorname{sign}\left(I_{Y}-h_{I}^{l} V_{I}^{m} I_{Y}\right)
\end{gathered}
$$

Also, to solve the line search problem in (9), we take the derivate of $F\left(I_{Y}\right)$ as follows,

$$
\frac{\partial F}{\partial S}=\frac{\partial F}{\partial\left(I_{Y}+S d\right)} \cdot \frac{\partial\left(I_{Y}+S d\right)}{\partial S}=d^{T} \cdot \frac{\partial F}{\partial\left(I_{Y}+S d\right)}
$$

According to (10), we know that

$$
\frac{\partial F}{\partial\left(I_{Y}+S d\right)}=-2 A^{\top}\left\{\left(G_{Y}-A\left(I_{Y}+S d\right)\right\}+\gamma H^{\prime}\left(I_{Y}+S d\right)\right.
$$


Set $d^{T} \cdot \frac{\partial F}{\partial\left(I_{Y}+S d\right)}=0$, and $S$ can be solved as

$$
S=\frac{d^{\top}\left[A^{\top}\left(G_{Y}-A I_{Y}\right)-0.5 \gamma H^{\prime}\left(I_{Y}+S d\right)\right]}{d^{\top} A^{\top} A d}
$$

It is clear from the above equation that $S$ still exists on both sides of (13). A general solution is to design an iterative process with $S$ and set a small value for initialization. Different initial values of $S$ make little difference to the calculated step size due to the large fidelity error at the beginning of the iteration. Nevertheless, because of the unideal quadratic property of the BID model, such as the red curves shown in Fig.1, employing the step size in (13) cannot generate a stable convergent solution. In reality, the initial input is generated by bi-cubic interpolation, which is with low sharpness and far from the final optimal solution. From (5), we note that the $L_{2}$ norm fidelity error is much larger to the BTV regularization error, and the fidelity term shares more weight in early iterations. Therefore, the total error becomes more quadratic, such as the part of the red curve after about 10 iterations shown in Fig.1. However, with the increasing of iterations, fidelity error has been corrected continuously as well as the restored image becomes sharp. That is, the regularization term shares a heavier weight, whereas the fidelity term has a lighter weight and the objective function increasingly non-quadratic, such as the red curve between 10 and 30 iterations, as shown in Fig.1. Besides, consider that the BTV regularization term is the sum of the $L_{1}$ norm errors, whose gradient change quickly at some points. Hence, (5) based general line search strategy cannot converge with an incorrect step size, which means equation (13) has to be modified to lead a more stable solution. Intuitionally, (13) is reformulated as

$$
S=\frac{d^{\top}\left[A^{\top}\left(G_{Y}-A I_{Y}\right)-0.5 \gamma H^{\prime}\left(I_{Y}+S d\right)\right]}{d^{\top} A^{\top} A d+\beta}
$$

Along with the increasing of ieration times, $\beta$ can prevent $S$ from becoming oversized. Then, we calculate the step size according to (14) and substitute it into the CGbased BID method. In the experiment, motion blur and defocus blur are designed to simulate the actual situation to illustrate the effectiveness of the method. The image baby from our synthetic dataset, which is shown in Fig.3 is adopted as an example. We process images with ranged values of $\beta$ and use root-mean-square error (RMSE) to evaluate the quality of the sharp image. The convergence performance estimated is depicted in Fig.1. 


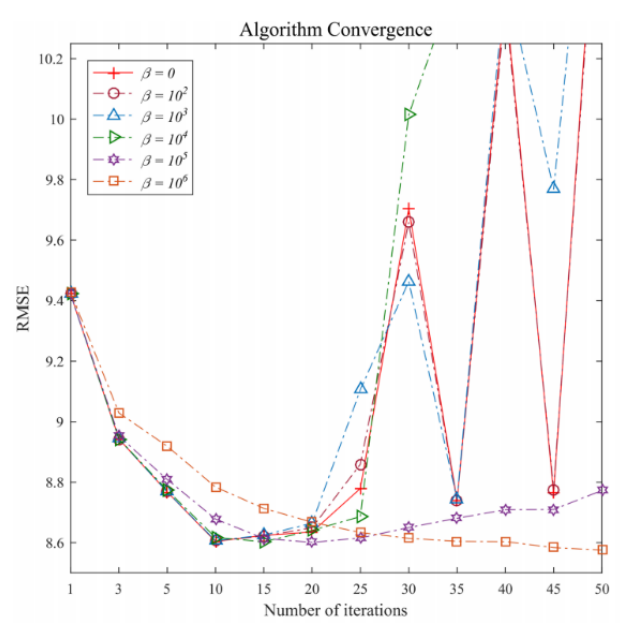

Fig. 1. RMSE of BID method with ranged step size.

From Fig.1 we note that the smaller $\beta$ is, the faster of algorithm convergence in the earlier stage of iterations, but it cannot stabilize the solution in a later period. On the contrary, a larger $\beta$ maintains the final solution stable, but convergence speed is slow. The 15 th iteration is used as the cut-off point. Before, it is monotonically decreasing for all $\beta$, but when the $\beta$ is smaller, RMSE decreases faster and the algorithm converges. For example, when $\beta=10^{6}$, its convergence rate is significantly lower than that when $\beta=$ $10^{2}$. After that, the smaller the beta, the more unstable it is and tends to diverge. Our ideal convergence tendency is to converge quickly in the early stage and to stabilize quickly in the later stage. Consequently, to have the fast convergence property and obtain a final stable solution, $\beta$ needs to change with iterations going on, and it should be proportional to the number of iteration. Conveniently, for illustrating the property of $\beta$, we introduce a parameter $\xi$ to express divergence degree, which is related to the $L_{2}$ norm of descent direction. It depends on the gradient difference between the last iteration and this iteration. The formulation of $\xi$ in the $j t h$ iteration is shown as

$$
\xi_{j}=\frac{d_{j 2}}{\sqrt{C * E}}
$$

where $d_{j}$ represents the descent direction of the estimated image in the $j$ th iteration, $C$ and $E$ represent row size and column size of $I$, respectively. The error is corrected continuously, and $\xi$ decreases simultaneously based on iterations. Inversely, as iteration goes on, it will lead to a divergence if $\xi$ becomes large. After the above analysis, judging the convergence condition by comparing divergence degree of two neighboring iterations, we defined, at each iteration $j$, a gradient-wise difference matrix $M_{g}$ as

$$
M_{g}(j)=\left|\xi_{j}-\xi_{j-1}\right|
$$


where $M_{g}$ represents the convergence degree in iterations. As mentioned previously, $\beta$ needs to increase with the iterations going on. To transform the convergence degree into a positive factor $\beta$, we introduce Gauss error function (ERF) as

$$
\beta_{\text {erf? }}=\frac{1}{2}\left(1-\int_{0}^{M_{g}(j)} e^{-\eta^{2}} d \eta\right)
$$

where $\eta$ is a scaler, $\beta_{\text {erf }}$ returns the integral of the corresponding interval. For emphasizing the characteristics of $\beta_{\text {erf }}$, several common monotonically increasing functions are used for comparison, including tanh function, sigmoid function, and two other general functions. We make some modifications to the formulation of six functions, as shown in Table I, and the curve of these positive factors is depicted in Fig.2. To facilitate comparison, we normalize all the modified functions and limit the range of all functions to $[0,1]$.

Table 1. Six positive functions with $\mathrm{Mg}$.

\begin{tabular}{lc}
\hline Positive factors & Functions \\
\hline$\beta_{\text {erf }}$ & $\frac{1}{2}\left(1-\int_{0}^{M_{g}} e^{-\eta^{2}} d \eta\right)$ \\
$\beta_{\text {sigmoid }_{1}}$ & $\frac{1}{1+e^{M_{g}}}$ \\
$\beta_{\text {sigmoid }_{2}}$ & $\frac{1-e^{-M_{g}}}{2}$ \\
$\beta_{\text {tanh }}$ & $\frac{1}{2}-\frac{M_{g}}{2 \sqrt{1+M_{g}{ }^{2}}}$ \\
$\beta_{\text {general }_{1}}$ & $\frac{1}{2}-\frac{1}{\pi} \arctan \left(\frac{\pi}{2} M_{g}\right)$ \\
$\beta_{\text {general }_{2}}$ &
\end{tabular}

From Fig.2 we note that $\beta_{\text {erf }}$ has a large value than the other four positive factors with small $M_{g}(j)$. Therefore, $\beta_{\text {erf }}$ has a fast convergence rate and high stability. Finally, we substituted $\beta_{\text {erf }}$ into (14) and get our proposed expression of step size as

$$
S_{j}=\frac{d_{j}^{\top}\left[A^{\top}\left(G_{Y}-A I_{Y(j)}\right)-0.5 \gamma H^{\prime}\left(I_{Y(j)}+S_{(j-1)} d_{j}\right)\right]}{0.5 d_{j}^{\top} A^{\top} A d_{j}+\left(1-\int_{0}^{M_{g}(j)} e^{-\eta^{2}} d \eta\right)}
$$

The whole procedure of the proposed gradient-wise step size search strategy is described in Algorithm 1, and Algorithm 2 is the pseudocode of we proposed algorithm.

\begin{tabular}{l}
\hline Algorithm 1 Gradient-wise Search Strategy. \\
\hline Input: $d_{j-1}, \xi_{j-1}, S_{j-1}, G_{Y}, I_{Y(j)} ;$ \\
1. Calculate $\xi_{j-1}$ according to (15); \\
\hline
\end{tabular}


2. Calculate $S_{j-1}$ according to (16) and (18);

Output: $\xi_{j}, S_{j}$.

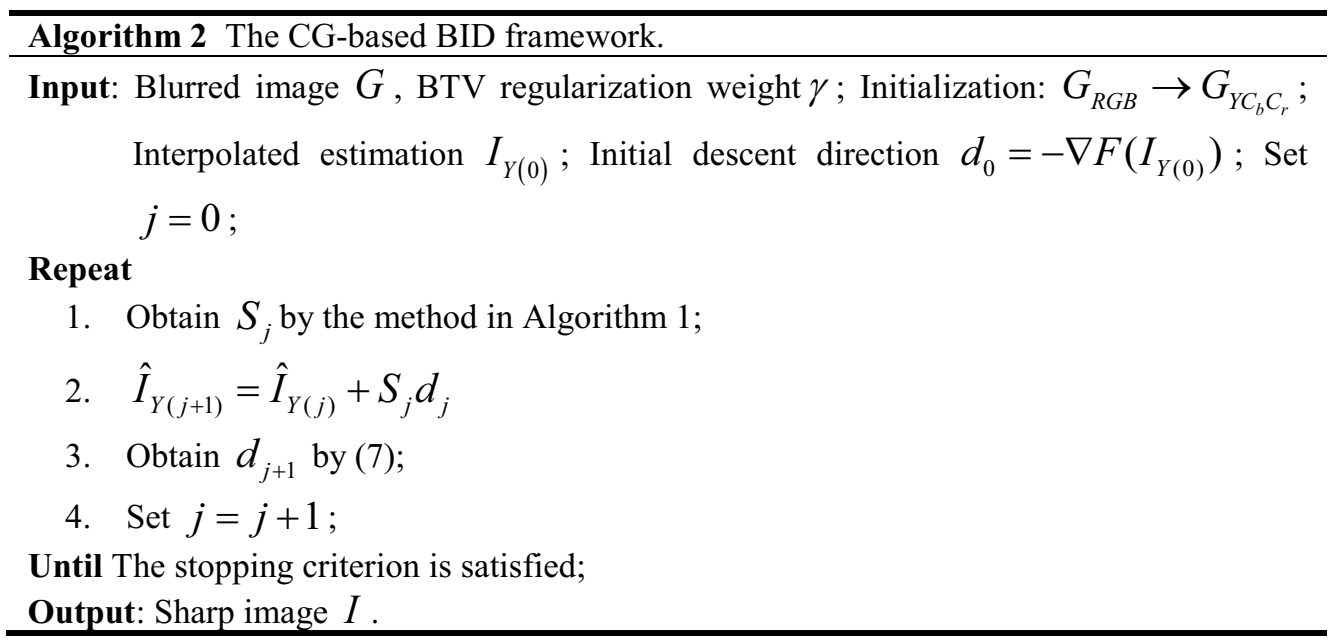

\section{Experiment results}

\subsection{Implementation details}

In this section, we introduce our implement details method ically including datasets and compared methods, parameters setting, and quantitative evaluation metrics. All experiments are coded in MATLAB R2016a and running on a Core i5-4210U @2.4 GHz CPU with a 4GB RAM. We conduct our experiment on our synthetic dataset, which is shown in Fig.3. The clear images from the dataset of Set5\&Set14 dataset, Pan's dataset [23] dataset and our real captured images.

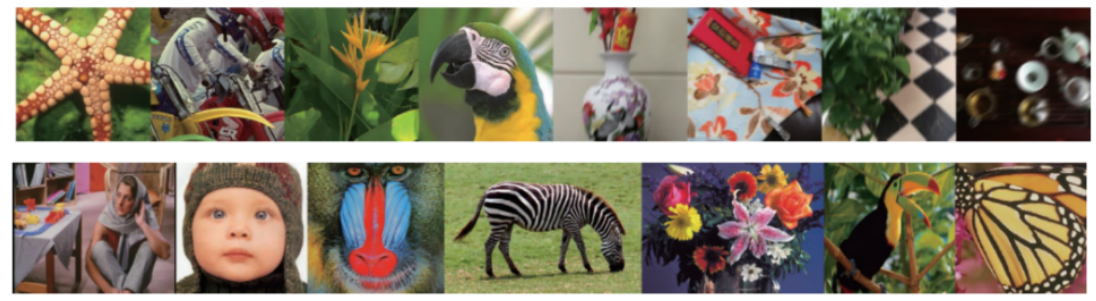

Fig. 3. Our dataset. 


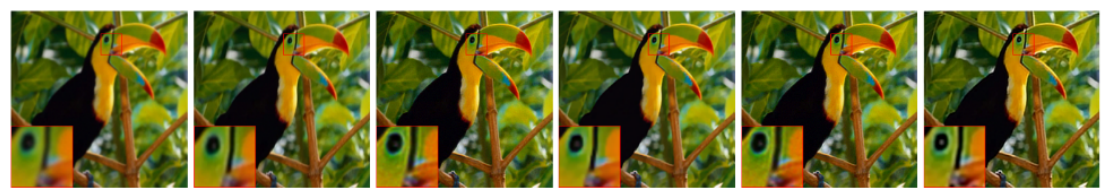

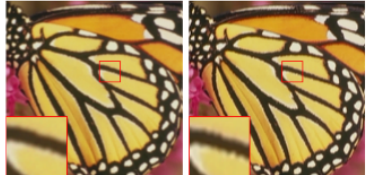

(a) Degradation

(b) Ours

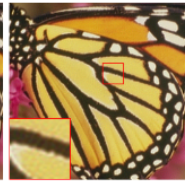

(c) Wen et al. [19]

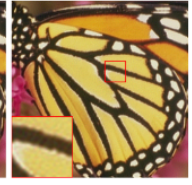

(d) Pan et al. [23]

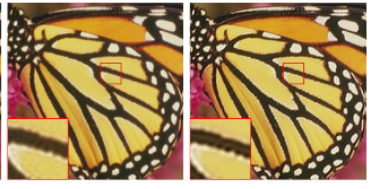

(e) Pan et al. [24] (f) Krishnan et al. [25]

Fig. 4. Comparison of deblurring results for defocus blurred image.

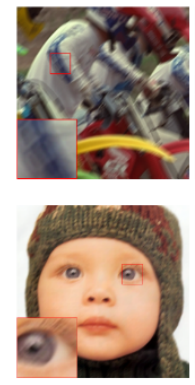

(a) Degradation
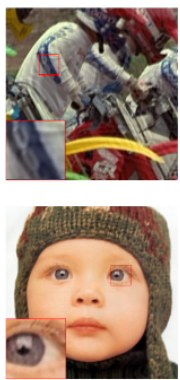

(b) Ours
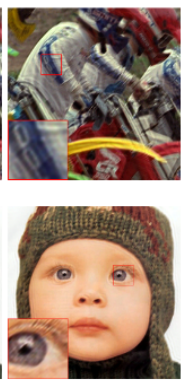

(c) Wen et al. [19]
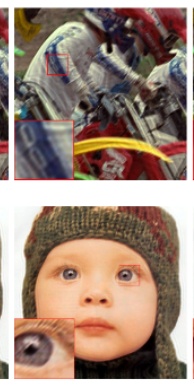

(d) Pan et al. [23]
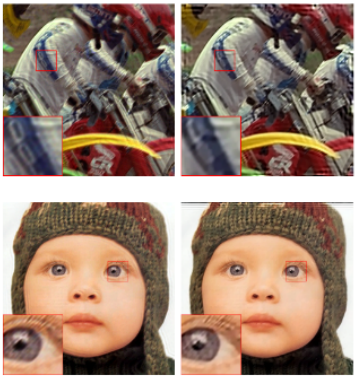

(e) Pan et al. [24] (f) Krishnan et al. [25]

Fig. 5. Comparison of deblurring results for motion blurred image.

The proposed scheme is compared visually and quantitatively with state-of-the-artschemes, including Wen et al. [19], Pan et al. [23], Pan et al. [24], Krishnan et al. [25] using different images. For synthetic scenes, the blurred image is generated and further adopted to estimated the sharp image. In the degrading process, warping the clear images by no more than 5 pixels, then the displacement deviation with $\mathrm{L}=10$, the angle of deviation with $\theta=$ 45 , to simulate the motion effect. Making a defocused blur image from the warped image that blurred by $5 \times 5$ Gaussian kernel with $\varrho_{g}=1$, which represents the defocus blurring. Finally, Gaussian noise with $\varrho_{n}=1.5$ is added. We employ BTV regularization terms to our proposed strategy that the parameter of BTV is the same as the value in [49]. Meanwhile, the BTV regularization weight with $\gamma=0.1$. To evaluate and compare the performance of estimated image quantitatively, root-mean-square error (RMSE), peak signal-to-noise ratio (PSNR), and mean structure similarity (SSIM) are employed.

\subsection{Results of synthetic images}

In our experiment, the number of iterations is fixed to 15 and compares the estimated results both visually and quantitatively. We first show the results of defocused image and motion blurred images processed by our method and other methods in Fig. 3 and Fig.4. It is seen that the red box region is the zoomed-in portion of the test image about three times larger, which can be observed that the proposed approach generates more sharp images than other algorithms while suppressing the ringing artifacts and eliminating additional noise. Among all of the competitive methods, the results show that the fidelity of our deblurred image is better than others. 
To make quantitative comparisons, we calculated the same quality index of different images and can see from the results that our method outperforms competing methods for quality metrics. Table.II shows the results of defocused image comparisons, and Table.III shows quantitative metrics of the motion blurred image.

Table 2. Quantitative comparisons for defocus blurred image.

\begin{tabular}{|c|c|c|c|c|c|c|}
\hline Image & Index & Krishnan et al. [25] & Pan et al. [24] & Pan et al. [23] & Wen et al. [19] & Ours \\
\hline \multirow{3}{*}{$\begin{array}{c}\text { toucan } \\
\left(288^{*} 288\right)\end{array}$} & RMSE & 15.36 & 12.16 & 9.57 & 8.81 & 6.93 \\
\hline & PSNR & 24.26 & 26.29 & 28.37 & 29.09 & 25.17 \\
\hline & SSIM & 0.8171 & 0.9039 & 0.7878 & 0.7748 & 0.9606 \\
\hline \multirow{3}{*}{$\begin{array}{c}\text { butterfly } \\
(256 * 256)\end{array}$} & RMSE & 23.79 & 16.31 & 25.34 & 23.38 & 24.31 \\
\hline & PSNR & 19.78 & 19.59 & 19.23 & 19.93 & 20.06 \\
\hline & SSIM & 0.7319 & 0.7221 & 0.7846 & 0.7457 & 0.8657 \\
\hline \multirow{3}{*}{ Average of sythetic dataset } & RMSE & 20.74 & 21.04 & 20.90 & 20.36 & 15.84 \\
\hline & PSNR & 21.56 & 21.62 & 21.84 & 20.48 & 20.99 \\
\hline & SSIM & 0.8156 & 0.7994 & 0.7612 & 0.7547 & 0.9307 \\
\hline
\end{tabular}

Table 3. Quantitative comparisons for motion blurred image.

\begin{tabular}{ccccccc}
\hline Image & Index & Krishnan et al. [25] & Pan et al. [24] & Pan et al. [23] & Wen et al. [19] & Ours \\
\hline rider & RMSE & 25.47 & 25.65 & 31.10 & 25.97 & $\mathbf{1 9 . 7 4}$ \\
$\left(256^{*} 256\right)$ & PSNR & 20.00 & 19.94 & 18.26 & 19.83 & $\mathbf{2 0 . 2 1}$ \\
& SSIM & 0.6014 & 0.5908 & 0.5012 & 0.5832 & $\mathbf{0 . 7 1 9 1}$ \\
\hline \multirow{2}{*}{ baby } & RMSE & 14.29 & 12.56 & 12.50 & 10.71 & $\mathbf{8 . 3 5}$ \\
$(512 * 512)$ & PSNR & 25.02 & 26.15 & 26.19 & 27.53 & $\mathbf{2 6 . 7 1}$ \\
& SSIM & 0.8829 & 0.8286 & 0.5901 & 0.8728 & $\mathbf{0 . 8 9 9 1}$ \\
\hline \multirow{2}{*}{ Average of sythetic dataset } & RMSE & 22.25 & 20.16 & 22.53 & 22.87 & $\mathbf{1 6 . 5 3}$ \\
& PSNR & 21.28 & 22.15 & 20.22 & 19.50 & $\mathbf{2 2 . 8 5}$ \\
& SSIM & 0.6395 & 0.6531 & 0.5901 & 0.6548 & $\mathbf{0 . 7 4 1 6}$ \\
\hline
\end{tabular}

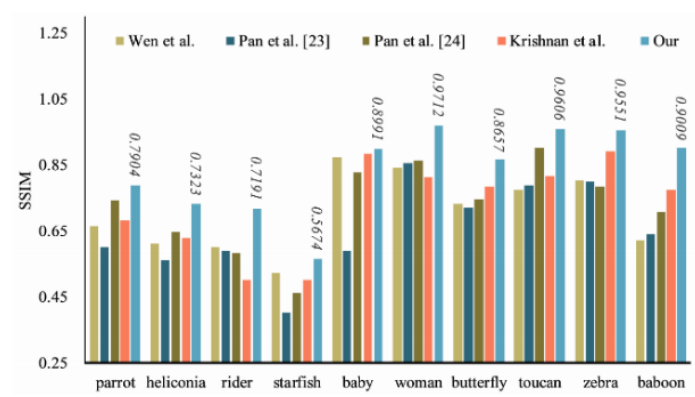

Fig. 6. Comparison of results in SSIM.

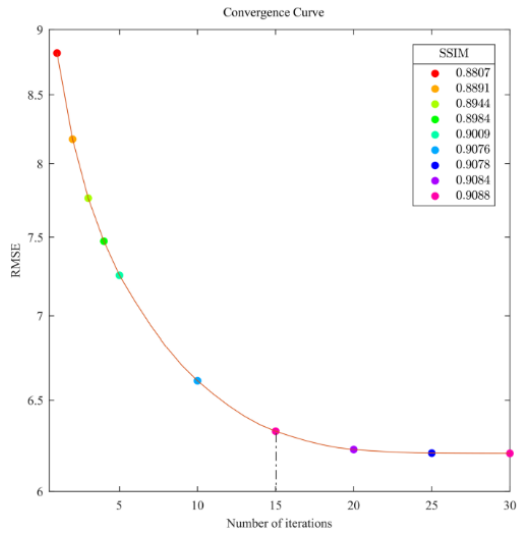

Fig. 7. RMSE and SSIM of scense Flower with ranged numbers of iterations. 


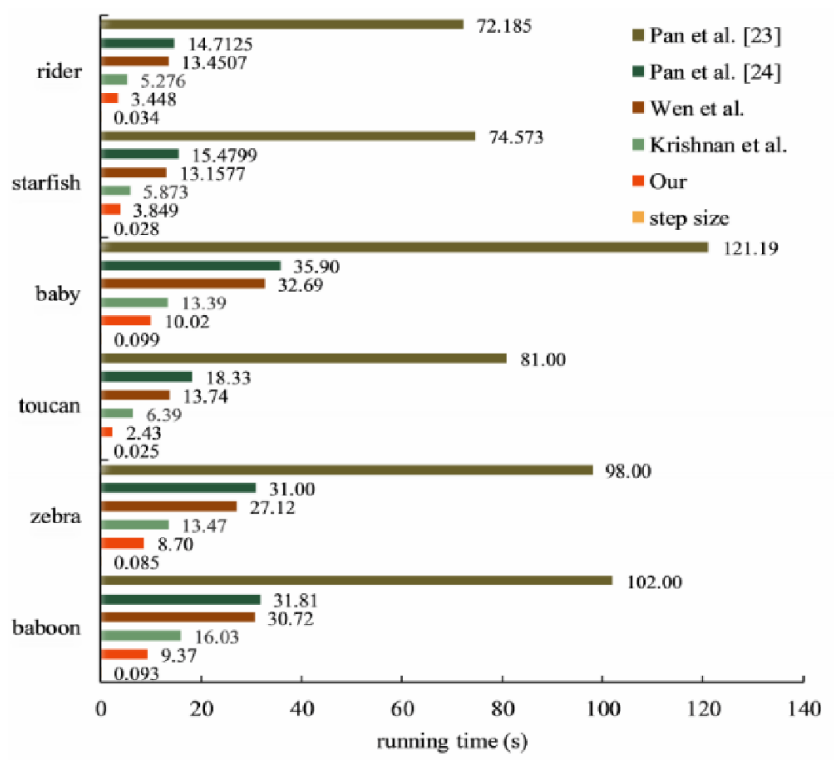

Fig. 8. Comparison of running times.
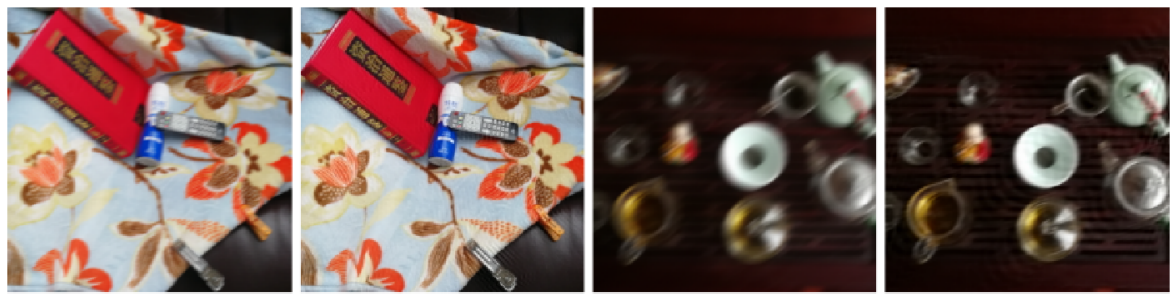

Fig. 9. Blind deblurring results for our real captured images.

Table.II and Table.III only list the specific data of the two images and the average value of the data set. We select the 10 images from the data set of SSIM and plot into a histogram for intuitive comparison, as shown in Fig.6. It is mentionable that our method reaches a higher precision among the synthetic dataset, obtains over 0.1 increase in SSIM compared with most other methods.

\subsection{Convengence performance}

We vary the number of iterations to study how the proposed method converges to the solution. RMSE and SSIM are regarded as the convergence indexes. What can be seen from Fig.7 is that our proposed method has a fast convergence performance with a remarkable decrease in RMSE and SSIM at 15 iterations. From Fig.8, for comparing the running time of each method, six images are selected from the synthetic dataset and processed in five methods. It is worth noting that the time used to calculate the step size is very little compared with the overall time of the algorithm, mainly because the step size does not design complex operations, but it can effectively accelerate the convergence of the algorithm. We notice that Pan et al. [23] needs the most time to process these images. On the contrary, our method has the least execution time. 


\subsection{Results of natural images}

The results of the proposed algorithm on two real natural images are shown in Fig.9. seen that the proposed gradient-wise search strategy helps to make accurate estimation, such as the Chinese characters on the cover of the red book, and the leaf. There are slight ringing artifacts around corners and edges. A main reason is that the real natural images often have saturated pixels which interfere with the iteration process.

\section{Conclusion}

This work have proposed a gradient-wise step size search strategy for adaptive iteration. It is simple yet effective in selecting suitable step size from blurred image to clear image. Further, modified CG method is used to obtain the global op timal solution of objective function with BTV regularization. Finally, extensive experiments on both specific and natural images demonstrated that it not only can achieve state-of-the art deblurring quality, but also can improve the computational efficiency under the CG-based framework. In brief, in terms of both the restoration quality and computational efficiency, the proposed algorithm is superior to all the other algorithms compared in this work.

This work was supported by the innovation capacity improvement project of Criminal Investigation Police University of China (grant No.2019YCZD05).

\section{References}

1. D. Gong, M. Tan, Y. Zhang, A. Van Den Hengel, Q. Shi, Self-paced kernel estimation for robust blind image deblurring, in: Proceedings of the IEEE International Conference on Computer Vision, 2017, pp. 1661- 1670.

2. X. Zhang, R. Wang, X. Jiang, W. Wang, W. Gao, Spatially variant defocus blur map estimation and deblurring from a single image, Journal of Visual Communication and Image Representation 35 (2016) 257-264.

3. L. He, Y. Wang, Z. Xiang, Support driven wavelet frame-based image deblurring, Information Sciences 479 (2019) 250-269.

4. X. Cai, Variational image segmentation model coupled with image restoration achievements, Pattern Recognition 48 (6) (2015) 2029-2042.

5. Q. Liu, L. Sun, Z. Shao, Nonblind image deblurring by total generalized variation and shearlet regularizations, Journal of Electronic Imaging 26 (5) (2017) 053021.

6. T. Li, H. Chen, M. Zhang, S. Liu, S. Xia, X. Cao, G. S. Young, X. Xu, A new design in iterative image deblurring for improved robustness and performance, Pattern recognition 90 (2019) 134-146.

7. D. Krishnan, R. Fergus, Fast image deconvolution using hyper-laplacian priors, in: Advances in neural information processing systems, 2009, pp. 1033-1041.

8. S. Wang, Z. Liu, W. Dong, L. Jiao, Q. Tang, Total variation based image deblurring with nonlocal self-similarity constraint, Electronics letters 47 (16) (2011) 916-918.

9. Y. Han, J. Kan, Blind color-image deblurring based on color image gradients, Signal Processing 155 (2019) 14-24.

10. Y. Bai, G. Cheung, X. Liu, W. Gao, Graph-based blind image deblurring from a single photograph, IEEE Transactions on Image Processing 28 (3) (2018) 1404-1418. 
11. J. Pan, J. Dong, Y.-W. Tai, Z. Su, M.-H. Yang, Learning discriminative data fifitting functions for blind image deblurring, in: Proceedings of the IEEE International Conference on Computer Vision, 2017, pp. 1068- 1076.

12. M. Ljubenovi'c, M. A. Figueiredo, Plug-and-play approach to class adapted blind image deblurring, International Journal on Document Analysis and Recognition (IJDAR) 22 (2) (2019) 79-97.

13. T. Michaeli, M. Irani, Blind deblurring using internal patch recurrence, in: European Conference on Computer Vision, Springer, 2014, pp. 783-798.

14. J. Zhu, K. Li, B. Hao, Hybrid variational model based on alternating direction method for image restoration, Advances in Diffference Equations 2019 (1) (2019) 34.

15. H. Yu, W. Wang, W. Fan, An adaptive iterative algorithm for motion deblurring based on salient intensity prior., TIIS 13 (2) (2019) 855-870.

16. K. Zhang, W. Zuo, Y. Chen, D. Meng, L. Zhang, Beyond a gaussian denoiser: Residual learning of deep cnn for image denoising, IEEE Transactions on Image Processing 26 (7) (2017) 3142-3155.

17. L. Ma, L. Xu, T. Zeng, Low rank prior and total variation regularization for image deblurring, Journal of Scientifific Computing 70 (3) (2017) 1336-1357.

18. J. Liu, M. Yan, T. Zeng, Surface-aware blind image deblurring, IEEE transactions on pattern analysis and machine intelligence.

19. F. Wen, R. Ying, P. Liu, T.-K. Truong, Blind image deblurring using patch-wise minimal pixels regularization, arXiv preprint arXiv:1906.06642.

20. J. Pan, R. Liu, Z. Su, X. Gu, Kernel estimation from salient structure for robust motion deblurring, Signal Processing: Image Communication 28 (9) (2013) 1156-1170.

21. X.-G. Lv, F. Li, T. Zeng, Convex blind image deconvolution with inverse filtering, Inverse Problems 34 (3) (2018) 035003.

22. Y. Liu, W. Lu, A robust iterative algorithm for image restoration, EURASIP Journal on Image and Video Processing 2017 (1) (2017) 53.

23. J. Pan, D. Sun, H. Pfifister, M.-H. Yang, Blind image deblurring using dark channel prior, in: Proceedings of the IEEE Conference on Computer Vision and Pattern Recognition, 2016, pp. 1628-1636.

24. J. Pan, Z. Hu, Z. Su, M.-H. Yang, Deblurring text images via $\mathrm{L}_{0}$ regularized intensity and gradient prior, in: Proceedings of the IEEE Conference on Computer Vision and Pattern Recognition, 2014, pp. 2901-2908.

25. D. Krishnan, T. Tay, R. Fergus, Blind deconvolution using a normalized sparsity measure, in: CVPR 2011, IEEE, 2011, pp. 233-240.

26. L. Xu, J. Jia, Depth-aware motion deblurring, in: 2012 IEEE International Conference on Computational Photography (ICCP), IEEE, 2012, pp. 1-8.

27. S. Zheng, L. Xu, J. Jia, Forward motion deblurring, in: Proceedings of the IEEE international conference on computer vision, 2013, pp. 1465-1472.

28. O. Whyte, J. Sivic, A. Zisserman, J. Ponce, Non-uniform deblurring for shaken images, International journal of computer vision 98 (2) (2012) 168-186.

29. D. Wipf, H. Zhang, Revisiting bayesian blind deconvolution, Journal of Machine Learning Research (JMLR).

30. S. Farsiu, M. D. Robinson, M. Elad, P. Milanfar, Fast and robust multiframe super resolution, IEEE transactions on image processing 13 (10) (2004) 1327-1344. 
31. E. S. Lee, M. G. Kang, Regularized adaptive high-resolution image reconstruction considering inaccurate subpixel registration, IEEE Trans actions on image processing 12 (7) (2003) 826-837.

32. R. C. Hardie, K. J. Barnard, J. G. Bognar, E. E. Armstrong, E. A. Watson, Highresolution image reconstruction from a sequence of rotated and translated frames and its application to an infrared imaging system, Optical Engineering 37 (1).

33. D. A. Sorrentino, A. Antoniou, Improved hybrid demosaicing and color superresolution implementation using quasi-newton algorithms, in: 2009 Canadian Conference on Electrical and Computer Engineering, IEEE, 2009, pp. 815-818.

34. L. Grippo, F. Lampariello, S. Lucidi, A nonmonotone line search technique for newton's method, SIAM Journal on Numerical Analysis 23 (4) (1986) 707-716. 